\title{
Allocentric and egocentric spatial processing in middle-aged adults at high risk of late-onset Alzheimer's disease : the PREVENT Dementia study
}

Karen Ritchie ${ }^{\star 1,2}$, Isabelle Carrière ${ }^{\star 1,2}$, David Howett ${ }^{3}$, Li Su $^{4}$, Michael Hornberger ${ }^{3}$, John T O’Brien ${ }^{4}$, Craig W Ritchie², Dennis Chan $^{3}$.

1. INSERM, University of Montpellier, Neuropsychiatry: Epidemiological and Clinical Research, Montpellier, France;

2. Centre for Dementia Prevention, University of Edinburgh, UK ;

3. Department of Clinical Neurosciences, University of Cambridge, UK;

4. Department of Psychiatry, University of Cambridge, Cambridge, UK

*Joint first authors

Corresponding author : Karen Ritchie, Inserm Unit1061: Neuropsychiatry, La Colombière Hospital, 39 Ave Charles Flahault, 34093 Montpellier Cedex 5 France.

karen.ritchie@inserm.fr

Keywords: cognition, neuropsychology, Alzheimer's disease, diagnosis, preclinical, MRI, prognosis

Funding : This work was supported by the Alzheimer's Society [grant numbers 178 and 264];

Alzheimer's Association [grant number TriBEKa-17-519007]; and philanthropic donations. 


\begin{abstract}
Impairments in spatial processing due to hippocampal degeneration have been observed in the years immediately preceding the diagnosis of Alzheimer's Disease (AD) dementia. The demonstration of changes in spatial processing in preceding decades would provide a cognitive marker for pre-clinical $A D$ and an outcome measure for early intervention trials. The present study examined allocentric and egocentric spatial processing in relation to future dementia risk in a middle-aged cohort. The CAIDE Dementia Risk Score (DRS) was calculated for 188 persons aged 40 to 59, of whom 94 had a parent with dementia. Participants underwent the Four Mountains Test (4MT) of allocentric spatial processing, the Virtual Reality Supermarket Trolley Task (VRSTT) of egocentric spatial processing and 3T MRI scans. A significant negative association was found between the DRS and 4MT (Spearman correlation $-0.26, p=0.0006$ ), but not with the VRSTT. The 4MT was also found to be a better predictor of risk than tests of episodic memory, verbal fluency or executive functioning. The results suggest that allocentric rather than egocentric processing may be a potential indicator of risk for late-onset $A D$, consistent with the hypothesis that the earliest cognitive changes in $A D$ are driven by tau-related degeneration in the medial temporal lobe rather than amyloid-only deposition in the medial parietal lobe.
\end{abstract}




\section{INTRODUCTION}

The universal failure to date of clinical trials aimed at slowing disease progression in patients with Alzheimer's disease (AD) related dementia has prompted increasing interest in the possibility of up-stream intervention at earlier, pre-dementia, phases of AD when treatments may be more effective. While biomarker studies suggest (AD) neuropathology to be present decades before the clinical diagnosis of dementia [1-3], the identification of functional markers, notably cognitive measures, indicative of incipient disease are more problematic. Such markers are, however, crucial for understanding the initial functional consequences of disease on the brain and for evaluating the effect of future treatments applied at the earliest stages of $A D$.

Theoretical models of disease progression have suggested an absence of early cognitive changes in the pre-clinical period [4] however these models have been based on small samples using cognitive tests recommended for later-stage dementia diagnosis. A recent review of neuroimaging and prospective epidemiological studies has suggested that preclinical alterations in cognitive function may exist, but detection would require the use of more sensitive and specific tests probing the function of brain regions first affected in AD [5]. As such, there is an increasing focus on the development of testing procedures suitable for the pre-clinical phase of $A D$ that evaluate the functions of the hippocampus and related structures, given that these are the first regions to exhibit tau-related neurodegeneration in addition to amyloid accumulation [6-7].

The known cognitive consequences of hippocampal cell loss are principally impairment in episodic memory and spatial information processing. Episodic memory has long been considered the central feature of cognitive impairment in AD dementia and although also observed in studies of pre-clinical AD [5], is not specific to hippocampal damage, but occurs in 
the context of damage to other brain regions such as the frontal lobes, and is also present in non-AD. By comparison, spatial processing in $A D$ has received relatively scant attention despite the discovery nearly 50 years ago that hippocampal neurons (place cells) exhibit spatially-modulated firing activity [8] and that place cell firing is coupled to spatial memory [9]. Functional neuroimaging has subsequently shown that the human hippocampus is also involved in spatial processing, as a core part of a network of brain regions which also includes the entorhinal cortex, retrosplenial cortex and precuneus [10-12]. Indeed, there is wellestablished strong structural and functional connectivity between these brain regions, suggesting that they might be an ideal neural and cognitive substrate for pre-clinical $A D$ changes [13].

Behavioural tests of spatial processing derived largely from cognitive and experimental psychology, have focused primarily on spatial orientation and memory in healthy persons and have only recently been applied to studies of hippocampal involvement in neurodegenerative disease. Bird et al. [14] found that performance on the Four Mountains Task (4MT) [15] of allocentric spatial memory was impaired in patients with early AD dementia, with a similar observation being made by Pengas et al. [16] in a study using several different tests of spatial memory. Crucially for the study of $A D$ in its earlier stages, Moodley et al. [17] found that performance on the $4 \mathrm{MT}$ was not only able to discriminate patients with mild cognitive impairment $(\mathrm{MCl})$ from age-matched controls, but was also able to differentiate $\mathrm{MCl}$ patients with and without cerebrospinal fluid (CSF) biomarker evidence of underlying AD . Notably, this differentiation of $\mathrm{MCl}$ groups at higher and lower risk of developing $\mathrm{AD}$ dementia using currently recognized biomarkers [18] was not achieved using the Rey Auditory Verbal Learning Test and the Trail Making Test both of which are considered sensitive to early AD and therefore included in the FDA-recommended Pre-Alzheimer Cognitive Composite battery for use in preclinical AD trials [20]. Impairment of allocentric spatial processing in people at risk of $A D$ dementia has also been shown with a variety of other tasks, ranging from the demonstration of reduced $\mathrm{fMRI}$ activation of the entorhinal cortex associated with altered navigational behaviour in young adult $A P O E$-e4 carriers [21] and deficient virtual way-finding in people with 
preclinical $A D$ [22] through to use of real world navigation tasks to identify spatial impairments in patients with $\mathrm{MCl}$ [23]). A systematic review across multiple methods has concluded that deficits of allocentric spatial processing occur from the earliest stages of $A D$ [24].

One complication, however, with allocentric spatial memory tests is that they rely on intact episodic memory processes to be completed. As outlined above, some of these tests have been shown to outperform existing non-spatial episodic memory tests, while others have not [16]. In particular, non-verbal episodic memory tests (eg. Rey Figure, PAL) have often shown to have similar sensitivity and specificity to spatial memory tests [16]. A different approach is to tap into egocentric spatial navigation processes, which rely much less on episodic memory intactness and targeting more specifically retrosplenial and precuneus brain areas [25]. The Virtual Reality Supermarket Trolley Task (VRSTT) [26] is, to our knowledge, the only clinically available test to tap into egocentric spatial orientation. The VRSTT has been shown to correlate with retrosplenial/precuneus atrophy in $A D$ and also distinguishes $A D$ from other neurodegenerative conditions with hippocampal atrophy (ie. bvFTD, sv-PPA) [26]. A more recent version of the VSRTT has also shown that egocentric and allocentric spatial orientation can be dissociated by the test [26], further highlighting the need for tests which tap into alloand egocentric processing to reliably differentiate spatial navigation deficits in preclinical and symptomatic $A D$.

Preliminary evidence thus suggests that tests of spatial processing may provide more sensitive outcome measures than currently used cognitive tests. However, the preclinical phase of $A D$ may cover several decades, and at present little is known on either the sensitivity of such tests to early brain changes during this period, or at what distance from dementia onset such change might first be detected. To test the hypothesis that spatial processing is affected in preclinical $A D$, the present study tested a cohort of 40-59 year old individuals at risk of developing late onset dementia within the pilot phase of the PREVENT Dementia cohort study [20]. Two types of spatial processing were examined: (i) personindependent allocentric processing, considered to be subserved primarily by the 
hippocampus, which relates to the encoding of information about the location of objects in relation to other objects external to the self, and (ii) person-dependent egocentric processing, primarily reflecting the function of medial parietal lobe regions [17], encoding the location of objects relative to the body axes of the self (left/right front/back up/down). Given that cognitive impairment is correlated with tau pathology, and that tau-related neurodegeneration is observed in the hippocampus prior to the medial parietal lobe, the study prediction was that increased dementia risk in the preclinical phase would be associated more with allocentric spatial processing than egocentric spatial processing. In this study dementia risk is defined according to a previously validated dementia risk score combining genetic and cardiovascular risk factors.

\section{MATERIALS AND METHODS}

\section{Subjects}

The PREVENT Dementia Programme is a prospective multi-site study of the adult children (40 to 59 years of age at recruitment) of persons with dementia, and an equal number of those without a parent with dementia who are in the same age band. The study is designed to identify clinical and biological changes in the pre-clinical phase of $A D$ which may subsequently be used as short-term outcome measures for mid-life secondary preventions. The protocol for the PREVENT Dementia study has been described in detail elsewhere [19].

For the purposes of the present research only the results from one PREVENT study site (West London) were used, as this is currently the only site for which spatial testing and genotyping have been completed for all participants. West London participants were recruited through multiple sources including_the dementia register (DemReg) database held at West London Mental Health NHS Trust (WLMHT), the Join Dementia Research website (https://www.joindementiaresearch.nihr.ac.uk/), and also by information about the study 
through the internet and public presentations. Of the 188 persons included, 175 (124 women, 51 men) had complete cognitive data, and of these 94 have at least one parent with a dementia diagnosis, referred to as the family history (FH sub-group). One hundred and thirty two participants were 50 years or over, and 43 under 50 .

\section{Consent and organisation of examinations}

Consented participants were seen at the West London Cognitive Disorders Treatment and Research Unit, where they were given a standardized neuropsychiatric interview and lifestyle questionnaires. Blood was taken for ApoE genotyping with all members of the research and clinical teams remaining blind to the result. Approval for the study was given by the NHS Research Ethics Committee, London, Camberwell St-Giles.

\section{(i) Allocentric processing}

Allocentric processing was evaluated using the Four Mountains Task (4MT) [15] using the spatial memory score rather than processing speed, the latter not being available for all participants. This test consists of computer-generated landscapes, each featuring four mountains of different shapes and sizes. Subjects are asked to look at a landscape image for 8 seconds. After a 2 second delay they are asked to identify the previous-viewed landscape, but seen from a different viewpoint, from a selection of four landscapes (ie three foil images) presented simultaneously in 2x2 configuration (see Figure). Participants are given 30 seconds to choose; a lack of response after this time is allocated a zero score. Non-spatial features (lighting, vegetation colour, cloud cover) of both target and foil landscapes are varied between presentation and testing, such that non-spatial features of the image cannot be relied on to solve the task. The test is presented on an electronic tablet (Apple iPad) and subjects respond by touching their selected image on the tactile screen. All participants undergo three practice trials in order to ensure comprehension of the task, with the test proper consisting of 15 trials. The final test score was calculated as the number of correctly identified landscapes (maximum score 15). 
(ii) Egocentric processing

In PREVENT Dementia it is assessed by the Virtual Reality Supermarket Trolley Task (VRST) [26] which involves presentation of 14 video vignettes within a simulated supermarket presented on a tablet (Apple iPad) and seen from a first person perspective. From the entrance participants follow a route through the supermarket which involves a series of $90^{\circ}$ turns after which the subject is required to point towards the entrance. The response is recorded according to a theoretical quadrant (upper and lower left, upper and lower right). The number of correct locations (left or right upper quadrant or left or right lower quadrant) are recorded as well as error type. The score range is $0-14$.

In order to compare the performance of these tests with other cognitive tests commonly used in preclinical and prodromal research, associations with DRS were also examined in relation to visual and verbal episodic memory (immediate and delayed recall of names and faces), executive functioning (progressive matrices test of visual reasoning) and lexical access (verbal fluency). These tests, taken from the COGNITO Battery and their administrative procedures within the PREVENT Dementia protocol are described in detail elsewhere [19].

\section{Imaging}

All participants underwent 3T MRI scanning on a single scanner (Siemans Verio) including whole brain T1-weighted scans (176 slices, $1.0 \times 1.0 \mathrm{~mm}, 1.0 \mathrm{~mm}$ slice thickness, TR = $2300 \mathrm{~ms}, \mathrm{TE}=2.98 \mathrm{~ms}$, flip angle $9^{\circ}$ ) up to two years before the cognitive testing. Brain tissue segmentation into grey matter (GM), white matter (WM) and cerebrospinal fluid (CSF) was performed using the Gaussian mixture model in VBM toolbox of SPM8 (http://www.fil.ion.ucl.ac.uk/spm). The GM maps were then normalized using the DARTEL algorithm [27]. Hippocampal region of interests (ROIs) were selected using AAL atlas in MNI space [28], and then inverse normalized back to each subject's native space using the participant-specific diffeomorphic parameters estimated from the previous DARTEL 
procedure. The resulting ROls were also masked using the thresholded GM probability maps (at threshold $p>0.8$ ) before the total hippocampal volume was calculated. To control for inter-individual differences in head size, the hippocampal volumes are given as a proportion of the estimated total intracranial volume (ICV).

\section{Genotyping}

Taqman Genotyping was carried out in a single laboratory on QuantStudio12K Flex to establish ApoE variants. Genomic DNA was isolated from whole blood and genotyping was performed in 384 well-plates, using the TaqMan polymerase chain reaction-based method. The final volume PCR reaction was $5 \mu \mathrm{l}$ using $20 \mathrm{ng}$ of genomic DNA, $2.5 \mu \mathrm{l}$ of Taqman Master Mix and $0.125 \mu$ l of 40x Assay By design Genotyping Assay Mix, or $0.25 \mu l$ of 20x Assay On Demand Genotyping Assay. The cycling parameters were $95^{\circ}$ for 10 minutes, followed by forty cycles of denaturation at $92^{\circ}$ for 15 seconds and annealing/extension at $60^{\circ}$ for 1 minute. PCR plates were then read on ThermoFisher QuantStudio 12K Flex Real Time PCR System instrument with QuantStudio 12K Flex Software or Taqman Genotyper Software v1.3.

\section{Statistical analyses}

Dementia Risk Scores (DRS) were calculated for each participant irrespective of family history of dementia using the CAIDE score [30]. This is a risk score validated within prospective population studies and demonstrated to predict dementia onset up to 40 years before diagnosis [30]. It is composed of cohort-based weightings by reference to the following variables: age, education, sex, systolic blood pressure, body mass index, total cholesterol, physical activity, ApoE status. These sub-scores were derived from the PREVENT clinical examinations in the same way as for the Kivipelto et al. study of mid-life prediction of dementia [29]. Scores vary from 0 to 15 and are treated as a continuous variable in the present analyses. 
Comparisons of sociodemographic characteristics and prevalent pathologies between $\mathrm{FH}$ and non $\mathrm{FH}$ groups were performed using Chi square tests for categorical variables and Wilcoxon two-sample tests for continuous variables. As Box Cox analyses were unable to establish potential non-linear distributions, linear regression models were used to compare the $4 \mathrm{MT}$ score with other participant characteristics, while unpaired Wilcoxon tests were used for the Supermarket Trolley test score owing to its highly skewed distribution. Comparisons of sociodemographic characteristics and prevalent pathologies between $\mathrm{FH}$ and non $\mathrm{FH}$ groups were performed using Chi square tests for categorical variables and Wilcoxon two-sample tests for continuous variables. Spearman correlation coefficients for continuous characteristics and unpaired Wilcoxon tests for categorical characteristics were used to compare the 4MT score and the supermarket test with other participant characteristics. In order to make a more direct comparison between the 4MT and Supermarket Trolley tests, as well as with other tests commonly used to test preclinical and prodromal persons, the DRS mean score was also compared with tertiles of cognitive tests from the COGNITO battery (immediate recall of a name list, delayed face recall, delayed name recall, progressive matrices, verbal fluency, allocentric and egocentric spatial performances) using one way analysis of variance.

\section{RESULTS}

The age, socio-economic status and gender distributions of the participants are given in Table 1, comparing those with and without a family history $(\mathrm{FH})$ of dementia

Persons with a family history of dementia were found to have a lower level of education, a higher prevalence of the ApoE $\varepsilon 4$ allele, and to have a significantly higher dementia risk score (DRS) than controls (mean (SD) of 7.10 (2.66) vs 5.85 (2.91), $\mathrm{p}=0.003$ ). No association was found between the CAIDE dementia risk score and total brain or hippocampal volume.

Table 1 here 
Associations between the two tests in relation to participant characteristics are given in Table 2 and score distributions in Figure 1.

Table 2 here

Figure 1 here

\section{Allocentric processing}

4MT scores were calculated as the sum of correct responses in participants without missing data . A significant positive association was found between 4MT scores and number of years of education (Spearman correlation $0.23 ; p=0.02$ ) and a significant negative association between 4MT and dementia risk score (DRS) (Spearman correlation -0.26; $p=0.0006$ ).

Table 2 here

Within a univariate linear regression model (Table 2) the 4MT score was found to be significantly associated with age, family history of dementia, years of education and the DRS, but not with gender and ApoE $\varepsilon 4$ status alone. Within a multivariate model adjusted for age and education, the association between 4MT score and family history became non-significant $(p=0.11)$. No association was found with history of head injury. On structural MRI imaging no association was found between 4MT score and either total brain volume or total hippocampal volume.

\section{Egocentric processing}

Supermarket test performance was calculated as the total number of correctly designated quadrants (Figure 2). Women were found to have lower scores than men (unpaired Wilcoxon test $\mathrm{p}=0.002$ ) and persons under 50 higher scores than those over 50 (unpaired Wilcoxon test $p=0.04$ ). A high positive association was also found with education (unpaired Wilcoxon test $=0.0003$ ). No significant association was found between test scores and the DRS, history 
of head trauma or family history, ApoE $\varepsilon 4$ status or structural MRI measures (total brain and total hippocampal volume).

Scatterplots showing the association (regression line and 95\% confidence band) between 4MT and the Supermarket Trolley test with the DRS are given in Figure 2.

Figure 2 here

Given the skewed distribution of the Supermarket Trolley test it was not possible to compare them within a multivariate model. We therefore divided the scores of both tests into tertiles for a more direct comparison of their performance in relation to the DRS. We also converted the scores of the COGNITO tests into tertiles to comparetheir association their association with DRS to that of the spatial tasks. The results are given in Table 3.

Table 3 here

\section{DISCUSSION}

Given that preclinical $A D$ neurodegeneration is observed at an early stage in the hippocampus and related medial temporal lobe (MTL) structures, the role of these regions in the processing of spatial information suggests that impaired performance on spatial tests may represent an early behavioural marker of AD-related brain changes. This is supported by longitudinal MRI studies of $A D$ showing that altered performance on visuospatial tasks predates MTL atrophy in preclinical cases [31].

Within this middle-aged cohort, $54 \%$ have a parent diagnosed with dementia. On the basis of research indicating an association between age of onset in parents and age of diagnosis in offspring in both early-onset AD [32] and late-onset cases [33-34], we previously estimated persons with a family history of dementia in this cohort to be at a mean distance from dementia diagnosis of 24 years (SD 7.5) [35]. This suggests any future cases to be within a pre-clinical period. Family history is, however, an insufficient basis for defining dementia risk, 
so for this study we have calculated the CAIDE dementia risk score for all participants, as this algorithm combining the principal genetic risk of an ApoE $\varepsilon 4$ allele with cardiovascular risk factors, has previously been found to predict dementia on average 36 years before diagnosis. The CAIDE score was also found to predict white matter hyperintensity volume and hippocampal volume on an average of 17 years before diagnosis [36]. While previous studies have shown a cross-sectional relationship between CAIDE dementia risk score and brain volume changes in the prodromal period [36], this was not observed in preceding decades [37].

Performance on the delayed match-to-sample 4MT of allocentric spatial processing was not associated with family history of dementia, or the ApoE e4 allele. However, a significant association was observed between 4MT score and the CAIDE dementia risk score, although the correlation coefficient was quite small. In contrast, no association was observed between performance on the VR Supermarket Trolley Test of egocentric spatial memory/processing and DRS, ApoE4, FH, brain or hippocampal volume. It is possible, however, that the positive skew of the egocentric processing test has given rise to lower sensitivity to mild decrements, as compared to the normal distribution of the allocentric test. Increases in cohort size over time may yield a less skewed distribution which would allow us to better examine this point.

Women were found to have a significantly lower score on egocentric processing than men, with no difference being observed for allocentric processing. This observation is coherent with previous studies suggesting that women tend to have more of a landmarkbased strategy while men utilise both landmark-based and global navigation strategies [37].

In order to make a more direct comparison of the two tests in relation to the risk score, we divided scores from both tests plus tests taken from the COGNITO battery commonly used in preclinical studies, into tertiles. Although this could potentially have lowered the association for $4 \mathrm{MT}$, we found that the differential discrimination of the two tests remained. No relationship was found between the CAIDE dementia risk score and either the Supermarket 
Trolley test, a test of immediate verbal recall, a progressive matrices test of executive functioning, delayed visual recall or verbal fluency. Only delayed verbal recall following interference tasks reached a low level of significance, whereas the 4MT showed a far stronger association.

At this time, estimated on average to be around 24 years prior to possible dementia diagnosis in persons with a familial risk, there was no evidence of an association between the CAIDE dementia risk score and structural changes in the total brain or total hippocampal volume. This is consistent with longitudinal findings from the CAIDE study [37].

Within a previous report on cognitive performance within this cohort carried out two years before, we found no association between commonly used tests of episodic memory and either the CAIDE dementia risk score or other dementia risk factors, although a test for recall of the relative location of objects, a test implicating, although not specific to, allocentric spatial memory, was found to be associated with total hippocampal volume [35].

Taken together, at this very early age and distance from possible dementia onset, our data indicate that allocentric, but not egocentric, spatial processing may be an important cognitive feature of risk for late onset AD. This is supportive of the study hypothesis and is consistent with theories of hippocampal function relating to its role in allocentric spatial processing [38]. These findings also align with the observations of Laukka et al. [39] who found that visuospatial deficits preceded changes in episodic and semantic memory for ten years before dementia diagnosis.

One tentative conclusion to be drawn at this stage, but one in line with our a priori hypothesis and prediction, is that a test based on the function of the hippocampus, one of the brain regions initially affected by tau-related neurodegeneration, is a more sensitive cognitive marker of future dementia risk than a test probing the function of medial parietal regions associated with early amyloid but not early tau deposition. This preliminary conclusion would be consistent with the hypothesis that it is the tau, not amyloid spread that best predicts 
cognitive decline in AD. An alternative explanation of the relationship between CAIDE and allocentric spatial processing may be that CAIDE is detecting vascular changes occurring more in the hippocampus than the medial parietal areas independently of AD processes, although recent research suggests that such changes may in themselves be triggering $A D$ tau accumulation independently of $A \beta[40]$.

These results represent the first set of data from one PREVENT Dementia study site, and will be followed up by completion of spatial tests for the entire PREVENT Dementia cohort across five sites (West London, Cambridge, Oxford, Edinburgh and Dublin), with attendant increases in the power of the study. If these findings are supported by larger scale testing, then they have significant implications for clinical and translational AD research. First, they would support the existing case for including allocentric spatial memory tests in cognitive batteries aimed at preclinical AD [41], both to aid stratification of at-risk individuals into interventional studies and to use as outcome measures to evaluate the effect of interventions aimed at this early stage of $A D$.

Two limitations of the present study are first the use of the CAIDE score as a proxy of AD risk given that very long term follow-up to clinical diagnosis is not feasible. This score may pick up other forms of dementia and not be specific to AD. Kivipelto et al. [29] found from prospective follow-up that $79 \%$ of the predicted cases were AD. A second limitation is its cross-sectional design, previous cross-sectional studies of pre-clinical AD having shown virtually no association between cognitive performance and brain changes [42-43]. Follow-up of the PREVENT Dementia cohort will permit examination of spatial memory performance across time and also in relation to not only brain volume but also functional changes and increases in amyloid load as well as associations with fixed and modifiable risks for neurodegeneration. Prospective data will allow us to develop longitudinal disease models and determine changes in test scores over time that are associated with increased dementia risk. Finally, the use of tests based on the discovery of hippocampal place cells in rodents provides a bridge between studies of $A D$ in mouse models, which use the Morris Water Maze 
and other spatial memory tests to measure pathology-associated behavior change and effect of pharmacological intervention, and studies of cognition in human populations. The identification of outcome measures to assess treatment effect that are comparable across preclinical and clinical trial phases will address one of the current major unresolved issues in $A D$ research.

Acknowledgements : Genotyping was carried out by Lee Murphy, Edinburgh Clinical Research Facility, University of Edinburgh. JOB, LS and DC are supported by the Cambridge NIHR Biomedical Research Centre.

Conflict of Interests : The authors have no conflict of interest to declare

\section{REFERENCES}

[1] Ritchie K, Ritchie CW, Yaffe K, Skoog I, Scarmeas N (2015) Is late-onset Alzheimer's disease really a disease of midlife? Alz Dem Trans 15: 122-30

[2] Villemagne VL, Pike KE, Chatelat GI, Ellis KA, Mulligan RS, Bourgeat P, et al. (2011) Longitudinal assessment of $A \beta$ and cognition in aging and Alzheimer disease. Ann Neurol 69: 181-92.doi:10.1002/ana.22248

[3] Ritchie K, Carriere I, Berr C, Amieva H, Dartigues JF, Ancelin ML, Ritchie CW (2016) The clinical picture of Alzheimer's disease in the decade before diagnosis: clinical and biomarker trajectories. J Clin Psychiatry doi:10.4088/JCP.15m09989

[4] Jack CR, Knopman DS, Jagust WJ, Petersen RC, Weiner MW, Aisen PS, et al. (2013) Tracking pathophysiological processes in Alzheimer's disease: an updated hypothetical model of dynamic biomarkers. Lancet Neurol 12: 207-16.doi:S14744422(12)70291-0 [pii] 10.1016/S1474-4422(12)70291-0 
[5] Mortamais M, Ash JA, Harrison J, Kaye J, Kramer J, Randolph C, Pose C, Albala B, Ropacki M, Ritchie CW, Ritchie K (2017) 'Detecting cognitive changes in preclinical Alzheimer's disease: a review of its feasibility Alzheimers Dement 13: 468-492 doi: 10.1016/j.jalz.2016.06.2365

[6] Rowe CC, Ng S, Ackerman U, Gong SJ, Pike K, Savage G (2007) Imaging beta amyloid burden in aging and dementia Neurology 68: 1718-1725

[7] Khan UA, Liu L, Provenzano FA, Berman DE, Profaci CP, Sloan R, Mayeux R, Duff KE, Small SA (2014) Molecular drivers and cortical spread of lateral entorhinal cortex dysfunction in preclinical Alzheimer's disease. Nat Neurosci 17: 304-311

[8] O’Keefe J, Dostrovsky J (1971) The hippocampus as a spatial map. Preliminary evidence from unit activity in the freely-moving rat. Brain Res 34:171-175.

[9] O'Keefe J, Speakman A (1987) Single unit activity in the rat hippocampus during a spatial memory task. Exp Brain Res 68:1-27

[10] Maguire EA, Frith CD, Burgess N, Donnett JG, O'Keefe J (1998) Knowing where things are parahippocampal involvement in encoding object locations in virtual largescale space. J Cog Neurosci 10: 61-76

[11] Burgess, N., Becker, S., King, J. A., O'Keefe, J (2001) Memory for events and their spatial context: models and experiments. Philosophical Transactions of the Royal Society of London Series B Biological Sciences 356: 1493e1503.

http://dx.doi.org/10.1098/rstb.2001.0948.

[12] Iaria, G., Chen, J., Guariglia, C., Ptito, A., \& Petrides, M (2007) Retrosplenial and hippocampal brain regions in human navigation: complementary functional contributions to the formation and use of cognitive maps. Eur J Neurosci 25 : 890e899. http://dx.doi.org/10.1111/j.1460-9568.2007.05371.x.

[13] Coughlan G, Laczó J, Hort J, Minihane A-M, Hornberger M. Spatial navigation deficits - the overlooked cognitive fingerprint for incipient Alzheimer pathophysiology? Nat Rev Neurol (in press) 
[14] Bird CM, Chan D, Hartley T, Pijnenburg YA, Rossor MN, Burgess N (2010) Topographical short-term memory differentiates Alzheimer's disease from frontotemporal lobar degeneration. Hippocampus 20:1154-1169

[15] Hartley T, Bird CM, Chan D, Cipolotti L, Husain M, Vargha-Khadem F, Burgess N (2007) The hippocampus is required for short-term topographical memory in humans. Hippocampus. 17:34-4

[16] Pengas, G., Patterson, K., Arnold, R. J., Bird, C. M., Burgess, N, Nestor, P. J (2010) Lost and found: bespoke memory testing for Alzheimer's disease and semantic dementia. J Alzheimers Dis 21(4) : 1347e1365

[17] Moodley K, Minati L, Contarino V, Prioni S, Wood R, Cooper R, D'Incerti L, Tagliavini F, Chan D (2015) Diagnostic differentiation of mild cognitive impairment due to Alzheimer's disease using a hippocampus-dependent test of spatial memory. Hippocampus. 25:939-51

[18] Ritchie C, Noel-Storr AH, Takwoingi Y, Flicker L, McShane R, Mason SE, Smailagic N (2014) Plasma and CSF A-beta for the longitudinal prediction of Alzheimer's disease dementia and other dementias in people with cognitive decline but no dementia. Cochrane Database Syst Rev. Jun

[19] Ritchie CW, Ritchie K (2012) The PREVENT Study: A prospective cohort study to identify mid-life biomarkers of late-onset Alzheimer's disease. BMJ Open http://bmjopen.bmj.com/cgi/content/full/bmjopen-2012-001893

[20] Donohue MC, Sperling RA, Salmon DP, Rentz DM, Raman R, Thomas RG, Weiner M, Aisen PS (2014) The preclinical Alzheimer cognitive composite: measuring amyloid-related decline. Australian Imaging, Biomarkers, and Lifestyle Flagship Study of Ageing; Alzheimer's Disease Neuroimaging Initiative; Alzheimer's Disease Cooperative Study. JAMA Neurol 71: 961-70. doi: 10.1001/jamaneurol.2014.803. 
[21] Kunz L, Schröder TN, Lee H, Montag C, Lachmann B, Sariyska R, Reuter M, Stirnberg R, Stöcker T, Messing-Floeter PC, Fell J, Doeller CF, Axmacher N (2015) Reduced grid-cell-like representations in adults at genetic risk for Alzheimer's disease. Science 23:430-3

[22] Allison S, Babulal GM, Stout SH, Barco PP, Carr DB, Fagan AM, Morris JC, Roe CM, Head D (2018) Alzheimer Disease Biomarkers and Driving in Clinically Normal Older Adults: Role of Spatial Navigation Abilities. Alzheimer Dis Assoc Disord. 32:101-106

[23] Mokrisova I, Laczo J, Andel R, Gazova I, Vyhnalek M, Nedelska Z, Levcik D, Cerman J, Vlcek K, Hort J (2016) Real-space path integration is impaired in Alzheimer's disease and mild cognitive impairment. Behav Brain Res. 307:150-8

[24] Serino S, Cipresso P, Morganti F, Riva G (2014) The role of egocentric and allocentric abilities in Alzheimer's disease: a systematic review. Ageing Res Rev 16: 32-44.

[25] Vann SD, Aggleton JP, Maguire EA (2009) What does the retrosplenial cortex do? Nat Rev Neurosci. $10: 792-802$.

[26] Tu S, Wong S, Hodges JR, Irish M, Piguet O, Hornberger M (2015) Lost in spatial translation. A novel tool to objectively assess spatial disorientation in Alzheimer's disease and frontotemporal dementia. Cortex 67:83-94

[27] Ashburner J (2007) A fast diffeomorphic image registration algorithm. Neurolmage 38:95-113.

[28] Tzourio-Mazoyer N, Landeau B, Papathanassiou D (2002) Automated Anatomical Labeling of Activations in SPM Using a Macroscopic Anatomical Parcellation of the MNI MRI Single-Subject Brain. Neurolmage 15:273-289. 
[29] Kivipelto M, Ngandu T, Laatikainen T, Winblad B, Soininen H, Tuomilehto J (2006) Risk score for the prevention of dementia risk in 20 years among middle-aged people: a longitudinal population-based study. Lancet Neurol 5: 735-741

[30] Exalto LG, Quesenberry CP, Barnes D, Kivipelto M, Biessels GJ, Whitmer RA (2014) Midlife risk score for the prediction of dementia four decades later. Alzheimers Dement. 10:562-570.

[31] Laakso MP, Hallikainen M, Hanninen T, Partanen K, Soininen H (2000) Diagnosis of Alzheimer's disease : MRI of the hippocampus vs delayed recall. Neuropsychologia 38: $579-584$

[32] Bateman RJ, Xiong C, Benzinger TLS, Fagan AM, Goate A (2012) Dominantly Inherited Alzheimer Network.Clinical and biomarker changes in dominantly inherited Alzheimer's disease. N Engl J Med. 795-804.

[33] Scarabino D, Gambina G, Broggio E, Pelliccia F, Corbo RM (2016) Influence of family history of dementia in the development of late-onset Alzheimer's disease. Am J Med Genet B Neuropsychiatr Genet 171:250-6.

[34] Saad M, Brkanac Z,Wijsman EM (2015) Family-based genome scan for age at onset of late-onset Alzheimer's disease in whole exome sequencing data. Genes Brain Behav 14: 607-617

[35] Ritchie K, Carrière I, Su L, O'Brien JT, Lovestone S, Wells K, Ritchie CW (2017) The midlife cognitive profiles of adults at high risk of late-onset Alzheimer's disease: The PREVENT study. Alzheimers Dement. 13:1089-1097. doi: 10.1016/j.jalz.2017.02.008

[36] Stephen R, Liu Y, Ngandu T, Rinne JO, Kemppainen N et al. (2017) Associations of CAIDE Dementia Risk Score with MRI, PIB-PET measures and cognition. $J$ Alzheimers Dis 59: 695-705

[37] Maguire EA, Burgess N, O'Keefe J (1999) Human spatial navigation : cognitive maps, sexual dimorphism,and neural substrates. Curr Opin Neurobiol 9: 171-177 
[38] Vuorinen M, Spulber G, Damangir S, Niskanen E, Ngandu T, Soininen H, Kivipelto M, Solomon A (2015) Midlife CAIDE dementia risk score and dementia-related brain changes up to 30 years later on magnetic brain imaging. J Alzheimers Dis 44: 93-101

[39] O'Keefe J, Nadel L (1978) The Hippocampus as a Cognitive Map. University Press, Oxford.

[40] Laukka EJ, Macdonald SW, Fratiglioni L, Bäckman L (2012) Preclinical cognitive trajectories differ for Alzheimer's disease and vascular dementia. $J$ Int Neuropsychol Soc 18: 191-199

[41] Kim HJ, Park S, Cho H, Jang YK, Lee JS et al. (2018) JAMA Neurol doi:10.1001/jamaneurol.2018.0975

[42] Ritchie K, Ropacki M, Albala B, Harrison J, Kaye J, Kramer J, Randolph C and Ritchie CW (2017) Recommended cognitive outcomes in preclinical Alzheimer's disease: Consensus statement from the European Prevention of Alzheimer's Dementia project. Alzheimers Dement 13 :186-195

[43] Besson FL, La Joie R, Doeuvre L, Gaubert M, Mezenge F, Egret S, et al. (2015) Cognitive and Brain Profiles Associated with Current Neuroimaging Biomarkers of Preclinical Alzheimer's Disease. J Neurosci 35: 10402-10411.doi:35/29/10402 [pii] 10.1523/JNEUROSCI.0150-15.2015

[44] Toledo JB, Bjerke M, Chen K, Rozycki M, Jack CR, Weiner MW, et al. (2015) Memory, executive, and multidomain subtle cognitive impairment: Clinical and biomarker findings. Neurology doi:10.1212/wnl.0000000000001738 


\begin{tabular}{|c|c|c|c|}
\hline & Non FH & $\mathrm{FH}$ & Chi2 \\
\hline & $N=81$ & $N=94$ & $p$ value \\
\hline & $\%$ & $\%$ & \\
\hline Gender (women) & 70.4 & 71.3 & 0.90 \\
\hline Race (white) & 92.6 & 88.3 & 0.34 \\
\hline Education $(n=174)$ & & & 0.008 \\
\hline post-graduate (6) & 40.0 & 24.5 & \\
\hline trade or technical skills college & 17.5 & 7.4 & \\
\hline Colleges/university & 25.0 & 40.4 & \\
\hline high school (3) & 17.5 & 27.7 & \\
\hline Hypertension* & 12.4 & 20.2 & 0.16 \\
\hline Depressive symptoms ${ }^{\dagger}$ & 18.5 & 16.0 & 0.65 \\
\hline Diabetes & 1.2 & 5.3 & 0.14 \\
\hline Head injury (loss of consciousness) & & & 0.76 \\
\hline 1-2 times & 32.1 & 36.2 & \\
\hline 3 times or more & 6.2 & 4.3 & \\
\hline \multirow[t]{3}{*}{ ApoE $\varepsilon 4$ allele $(n=208)$} & 30.9 & 46.7 & 0.03 \\
\hline & median (IQR) & median (IQR) & Wilcoxon test \\
\hline & & & $p$-value \\
\hline Age (years) & $54.2(47.4-58.2)$ & $55.3(51.7-58.3)$ & 0.24 \\
\hline Body mass index $\left(\mathrm{kg} / \mathrm{m}^{2}\right)$ & $26.1(23.4-29.9)$ & $27.6(23.7-31.6)$ & 0.20 \\
\hline \multicolumn{4}{|c|}{ * hypertension: treated or blood pressure $>140 / 90 \mathrm{mmHg}$} \\
\hline
\end{tabular}


TABLE 2: associations of the Four Mountain score and the Supermarket test score with participant characteristics, $\mathrm{n}=175$

\begin{tabular}{|c|c|c|c|c|c|c|}
\hline & \multicolumn{3}{|c|}{ Four Mountain test } & \multicolumn{3}{|c|}{ Supermarket Test } \\
\hline & $\mathbf{N}$ & $\begin{array}{c}\text { Spearman correlation } \\
\text { coefficient }\end{array}$ & P value & $\mathbf{N}$ & $\begin{array}{c}\text { Spearman correlation } \\
\text { coefficient }\end{array}$ & P value \\
\hline Age (years) & 175 & -0.19 & 0.01 & 172 & -0.05 & 0.52 \\
\hline Years of education & 175 & 0.22 & 0.004 & 172 & 0.27 & 0.0003 \\
\hline Dementia risk score & 169 & -0.25 & 0.0009 & 166 & -0.02 & 0.78 \\
\hline Brain/ ICV in \% & 167 & 0.14 & 0.07 & 164 & 0.01 & 0.90 \\
\hline Hippocampus/ ICV in \% & 167 & 0.02 & 0.76 & 164 & -0.03 & 0.71 \\
\hline \multirow[t]{2}{*}{ Blows to head with LOC } & 175 & 0.004 & 0.96 & 172 & -0.04 & 0.59 \\
\hline & & Median (IQR) & P value* & & Median (IQR) & P value* \\
\hline Gender & 175 & & 0.05 & 172 & & 0.005 \\
\hline Men & & $11(8-12)$ & & & $11(10-12)$ & \\
\hline Women & & $10(7-12)$ & & & $10(8-12)$ & \\
\hline $\mathrm{FH}$ of dementia & 175 & & 0.05 & 172 & & 0.62 \\
\hline No & & $10(8-12)$ & & & $11(9-12)$ & \\
\hline yes & & $10(7-11)$ & & & $10(8-12)$ & \\
\hline
\end{tabular}


ApoE $\varepsilon 4$

No

Yes
172

$10(8-12)$

$10(8-11)$
0.27

170

0.47

*Wilcoxon two-sample test

ICV: Intra cranial volume, LOC: loss of consciousness 


\begin{tabular}{|c|c|c|c|}
\hline & DRS mean (SD) & $\begin{array}{l}\text { P value } \\
\text { One way } \\
\text { ANOVA }\end{array}$ & $\begin{array}{l}2 \text { by } 2 \\
\text { comparisons* }\end{array}$ \\
\hline \multirow[t]{2}{*}{$\begin{array}{l}\text { Face Recall, (number of faces correctly } \\
\text { recognised), } n=166\end{array}$} & & 0.82 & \\
\hline & $\begin{array}{l}6.72(0.41) \\
6.58(0.32) \\
6.34(0.44)\end{array}$ & & \\
\hline \multirow[t]{2}{*}{$\begin{array}{l}\text { Name Recall, (number of correct answers), } \\
n=166\end{array}$} & & 0.12 & \\
\hline & $\begin{array}{l}7.23(0.43) \\
6.49(0.30) \\
5.91(0.47)\end{array}$ & & \\
\hline $\begin{array}{l}\text { Name Recall, (number of names correctly } \\
\text { recalled), } n=166\end{array}$ & & 0.04 & $\begin{array}{l}\text { T1 vs T3 } \\
\mathrm{p}=0.03\end{array}$ \\
\hline $\mathrm{T} 1$ & $7.33(0.42)$ & & \\
\hline $\mathrm{T} 2$ & $6.61(0.35)$ & & \\
\hline T3 & $5.92(0.36)$ & & \\
\hline Matrix, (number of correct answers), $n=166$ & & 0.23 & \\
\hline $\mathrm{T} 1$ & & & \\
\hline $\mathrm{T} 2$ & $7.00(0.35)$ & & \\
\hline T3 & $\begin{array}{l}6.59(0.50) \\
6.15(0.33)\end{array}$ & & \\
\hline Matrices, (total time), $\mathrm{n}=166$ & & 0.46 & \\
\hline $\mathrm{T} 1$ & $6.75(0.38)$ & & \\
\hline $\mathrm{T} 2$ & $6.75(0.37)$ & & \\
\hline
\end{tabular}


Verbal fluency, (number of correct answers),

$\mathrm{n}=166$

T1

T2

$6.80(0.40)$

$6.29(0.34)$

T3

$6.68(0.41)$

Four Mountain score, $n=169$

$<0.0001 \quad$ T1 vsT3

T1

$7.33(0.37)$

T2

$6.88(0.33)$

T2 vs T3

$\mathrm{p}=0.002$

T3

$5.08(0.39)$

Supermarket score, $\mathrm{n}=166$

0.79

T1

$6.64(0.37)$

$\mathrm{T} 2$

$6.29(0.36)$

T3

$6.52(0.38)$

* Bonferroni adjusted $\mathrm{p}$-values

DRS: Dementia risk score

SD: standard deviation, $\mathrm{T} 1$ first tertile, $\mathrm{T} 2$ second tertile, $\mathrm{T} 3$ third tertile. 
Figure 1: Distribution of the Four Mountain Score $(n=175)$ and Supermarket Trolley score $(n=172)$
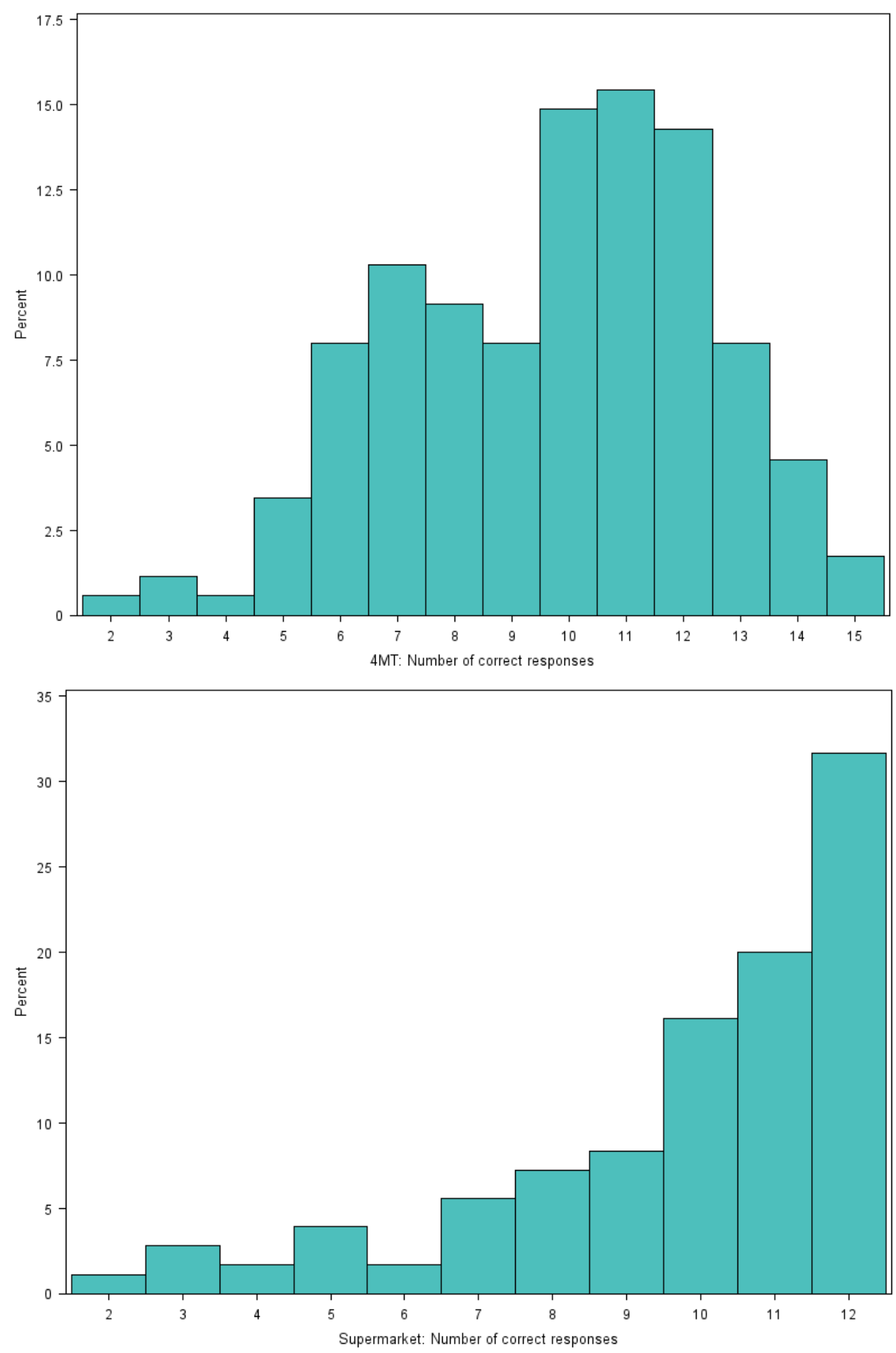
Figure 2: Scatterplot of the association of the Four Mountain (4MT) score and Supermarket Trolley score and the Dementia risk score (DRS) with the regression line and $95 \%$ confidence band
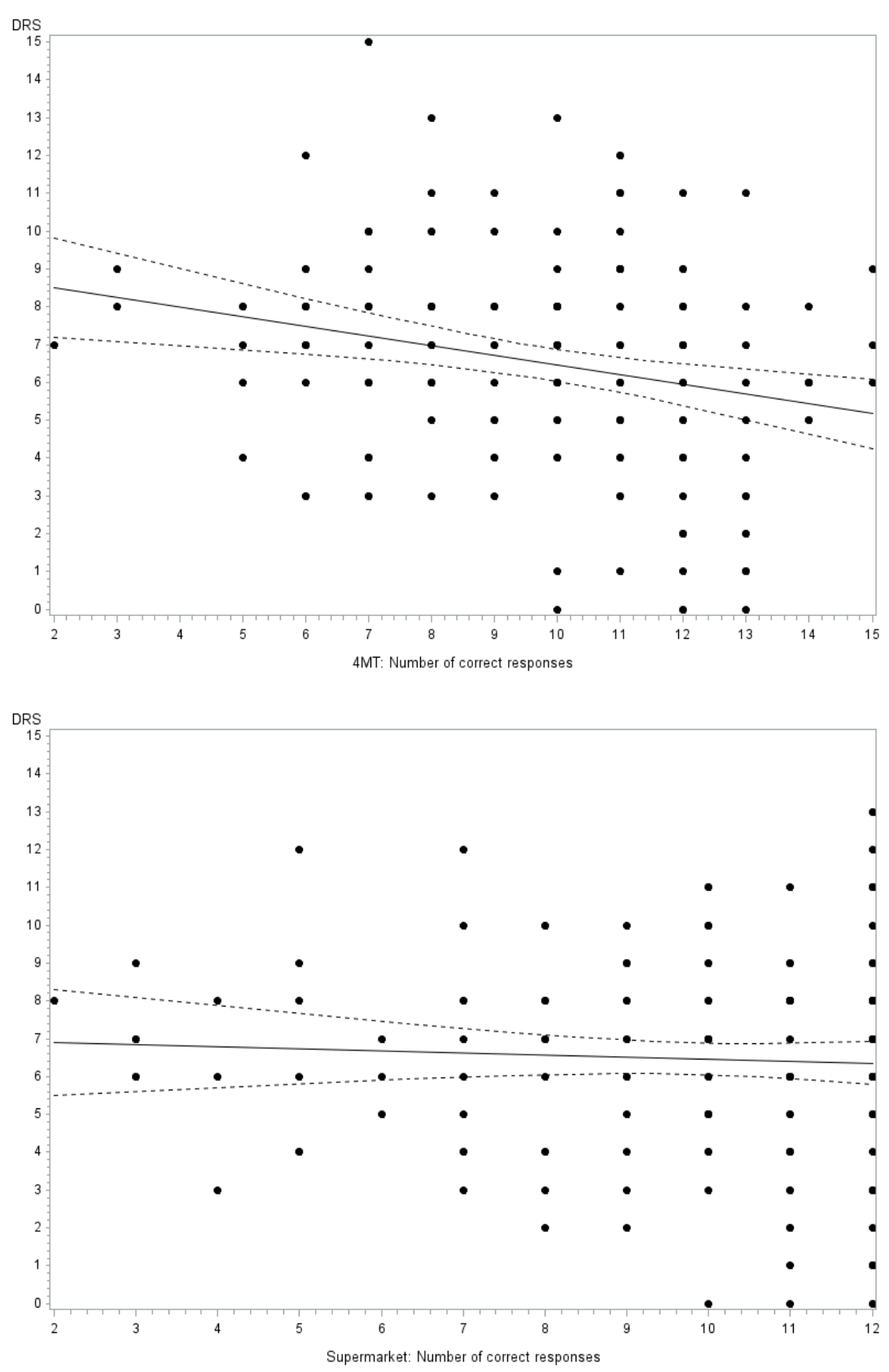